\title{
Valoración física, condición física y calidad de vida en pacientes con diferentes tratamientos renales sustitutivos
}

\author{
Sonsoles Hernández Sánchez¹, David García López², Alejandro Santos Lozano³, Gustavo González-Calvo, \\ Mónica Brazález Tejerina ${ }^{5}$, Nuria Garatachea Vallejo ${ }^{6}$ \\ ${ }^{1}$ Grupo de Investigación PROFITH. Departamento de Educación Física y Deportiva, Facultad de Ciencias \\ del Deporte, Universidad de Granada. España. 'Laboratorio de Fisiología, Facultad de Ciencias de la Salud, \\ Universidad Europea Miguel de Cervantes. Valladolid. España. ${ }^{3}$ Hospital Universitario 12 de Octubre. Madrid. \\ ${ }^{4}$ Departamento de Didáctica de la Expresión musical, plástica y corporal. Universidad de Valladolid. ${ }^{5}$ Enfermera. \\ Valladolid. ${ }^{6}$ Departamento de fisioterapia y enfermería, Facultad de Ciencias de la Salud y del Deporte, Universidad \\ de Zaragoza. Huesca. España
}

\section{Resumen}

La actividad física es de vital importancia en pacientes con enfermedad renal crónica, ya que mejora la capacidad funcional de los sujetos e incrementa notablemente su calidad de vida. Para mejorar la calidad de vida en pacientes con tratamiento renal sustitutivo, dializados o trasplantados, deberían ser incluidos programas de actividad física planificados y supervisados por profesionales. Existen varios estudios sobre entrenamiento aeróbico y de fuerza en pacientes con enfermedad renal crónica en hemodiálisis pero pocos sobre la condición física relacionada con la salud en estos pacientes y escasos los referentes a condición física en trasplantados y en pacientes con diálisis peritoneal. EI objetivo principal del estudio fue evaluar el nivel de actividad física, condición física y calidad de vida de enfermos renales crónicos con diferentes tratamientos sustitutivos.

Material y métodos: 25 enfermos renales crónicos varones de entre 59 y 72 años, que participaron voluntariamente en este estudio, fueron divididos en 3 grupos: trasplantados renales: $\mathrm{N}=11$, hemodializados: $\mathrm{N}=6$, y en tratamiento de diálisis peritoneal: $\mathrm{N}=8$. Se les administró el cuestionario de cuantificación de actividad física YALE, el cuestionario de calidad de vida KDQoL y realizaron la batería de tests Senior Fitness Test (SFT).

\section{Correspondencia: \\ Sonsoles Hernández Sánchez \\ C/. Martínez de la Rosa, 2. \\ 07180 Santa Ponça. Islas Baleares. \\ E-mail:sonsohs@gmail.com}

Resultados: No se encontraron diferencias significativas entre grupos en ninguno de los cuestionarios ni en los tests realizados. Los 3 grupos mostraron valores inferiores que otras poblaciones sanas de referencia de la misma edad.

Conclusiones: El tipo de tratamiento sustitutivo en los pacientes con enfermedad renal crónica no influye sobre el nivel de actividad física, condición física ni calidad de vida.

PALABRAS CLAVE

- ENFERMEDAD RENAL CRÓNICA

- CALIDAD DE VIDA

- ACTIVIDAD FÍSICA

- CONDICIÓN FÍSICA

Physical assessment, fitness and quality of life in patients with different renal replacement therapies

\begin{abstract}
Introduction: Physical activity has been shown very helpful in chronic renal patients (CKD) because it improves the subject's physical fitness and therefore their quality of life. To improve the quality of life in patients with substitute renal treatment, dialysated or transplanted, they should be included in physical activity programmes carefully planned and supervised by experts and professionals. There are several researches in literature about aerobic and strength training with patients with CKD in hemodialysis, but a few about physical condition related to health in this patients, and less than a few, the related researches about the physical condition in transplanted and
\end{abstract}


peritoneal dialysis patients. This study aimed to assess the physical activity, physical fitness and quality of life levels in chronic renal patients with different alternative treatments.

Material and methods: 25 male chronic renal patients aged between 59 and 72 years old were divided in 3 groups: renal transplant $(T x): N=11$, hemodialysis (HD): $N=6$, and peritoneal dialysis (DP): $N=8$. The YALE physical activity survey was administrated along with KDQoL questionnaire of quality of life. Furthermore, subjects performed the Senior Fitness Test (SFT).

Results: Significant differences between groups were not found concerning neither the questionnaires nor the physical tests. The three experimental groups showed lower values than same-age healthy individuals regarding both, the questionnaires and the physical tests.

Conclusion: Alternative treatments in ERC do not have influences on the level of physical activity, fitness and quality of life.

\section{KEYWORDS}

- CHRONIC RENAL PATIENTS

- QUALITY OF LIFE

- PHYSICAL ACTIVITY

- PHYSICAL FITNESS

\section{Introducción}

La evidencia científica demuestra que la realización de actividad física regular y apropiada se configura como el medio más eficaz para fomentar la salud y el bienestar de las personas. Así, un estilo de vida físicamente activo proporciona una protección parcial ante algunas enfermedades importantes de carácter crónico, entre las que se incluye la insuficiencia renal ${ }^{1}$. Son muchas las razones por las que se debe promover un régimen de ejercicio físico regular y apropiado entre la población mayor. Así, a nivel fisiológico y biológico está comprobado que hay una mejora de los órganos internos y de las capacidades físicas: disminución de la tensión arterial en reposo, control de la hipertensión arterial, un mayor control de la diabetes disminuyendo las necesidades de insulina y un mayor aumento del gasto calórico controlando la obesidad. También existe un cambio en los hábitos higiénico-dietéticos ya que se vuelven más saludables, y el ejercicio tiene un efecto relajante que ayuda a reducir el estrés ${ }^{2}$.

Los pacientes con enfermedad renal crónica (ERC) que reciben tratamiento de hemodiálisis (HD) y diálisis peritoneal (DP) tienen una considerable disminución en la tolerancia al ejercicio, en la capacidad funcional, en la resistencia y en la fuerza. Además, sufren una mayor pérdida de masa muscular que los sujetos sanos o pacientes con insuficiencia renal crónica severa que todavía no necesitan tratamiento sustitutivo renal ${ }^{3,4}$. La reducción del repertorio motor, junto a la lentitud de los reflejos, el descenso del tono muscular y la falta de capacidad funcional, entre otros factores, desembocan en un estado de inmovilidad e inactividad que agravan el envejecimiento y la incapacidad. No obstante, existen investigaciones que han demostrado que existe una reducción de la aparición de la fatiga y mejora en los niveles de función física con un incremento en los niveles de actividad física ${ }^{5}$. Por otra parte, a pesar de que existen ciertas controversias entre los resultados derivados de los diferentes cuestionarios de calidad de vida aplicados a pacientes con ERC, los factores físicos, psicosociales, sociodemográficos y clínicos parecen tener un efecto significativo ${ }^{6}$.

La capacidad funcional y la salud, relacionada con la calidad de vida, de los pacientes con ERC son inferiores en comparación con las de los individuos sanos como consecuencia del bajo nivel de actividad física ${ }^{7}$. Durante los últimos treinta años varias investigaciones han demostrado la necesidad de que los pacientes en HD deben realizar entrenamiento físico para alcanzar una mayor eficiencia cardiorrespiratoria ${ }^{1,8,9,10,11}$ rendimiento físico ${ }^{7,12}$ y una mejor calidad de vida relacionada con la salud, que en estos individuos es muy deficiente ${ }^{7,13,14,15}$. Hay muchos estudios en la literatura sobre entrenamiento físico en pacientes en HD, pero son escasos los referidos a la condición física y aún menos los referidos a la condición física en trasplantados y en pacientes con DP.

Por tanto, el objetivo del presente estudio ha sido estudiar y comparar el nivel de actividad física, condición física y calidad de vida en enfermos renales crónicos sometidos a HD, DP o trasplante (Tx). Como hipótesis de partida consideramos que los pacientes en diálisis obtendrían valores inferiores a los Tx en todas las pruebas realizadas. 


\section{Material y Métodos}

\section{Sujetos}

Se realizó una selección no aleatoria de 25 sujetos varones que participaron voluntariamente en este estudio con una media de $63,1 \pm 3,7$ años, $74,8 \pm 9,6$ $\mathrm{kg}$, y $1,6 \pm 0,0 \mathrm{~m}$ de altura. Todos ellos llevaban una

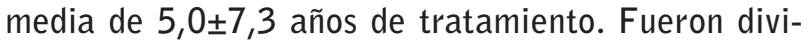
didos en tres grupos experimentales, en función del tipo de tratamiento sustitutivo: trasplantados renales ( $T x$ ): $N=11$, hemodiálisis (HD): $N=6$, en tratamiento de diálisis peritoneal (DP): $N=8$. Se contactó con todos ellos por medio de varios hospitales de Castilla y León. Los criterios de inclusión fueron: ser varón, de edad comprendida entre 60 y 70 años sometidos a tratamiento sustitutivo renal desde al menos 3 meses. Los criterios de exclusión fueron: haber padecido isquemia cardiaca o enfermedad severa coronaria en los últimos 6 meses, padecer enfermedades cardiorrespiratorias que impidiesen la realización de las pruebas y/o padecer afección cognitiva. Además del consentimiento informado de cada sujeto, se obtuvo un consentimiento médico de los facultativos de los correspondientes hospitales para participar en el estudio. Se respetó la Declaración de Helsinki.

\section{Diseño experimental}

Todas las evaluaciones se llevaron a cabo en horario de tarde y en días interdiálisis para los hemodializados. Cada sujeto asistió al laboratorio un solo día, donde se le aplicó en primer lugar el cuestionario de actividad física YALE, posteriormente el cuestionario KDQoL y por último los test físicos.

\section{Cuestionario YALE}

Para cuantificar la cantidad de actividad física realizada por los sujetos se administró el cuestionario YALE, desarrollado por Dipietro ${ }^{16}$ y validado al español ${ }^{7}$, para aplicarlo a personas mayores de 60 años. El YALE se divide en dos secciones: en la primera sección, muestra un trabajo físico, ejercicio y recreación de una lista de actividades para evaluar el tiempo empleado en este tipo de actividades durante una semana normal en el mes pasado. Las actividades recreativas van desde actividades de baja intensidad, tales como labores de punto, hasta actividades más intensas como el tenis. El tiempo dedicado a cada actividad se multiplica por un código de intensidad $\left(\mathrm{min} \cdot \mathrm{Kcal}^{-1}\right)$ y después se suman todas las actividades para crear un índice de gasto de energía semanal ( $\left.\mathrm{Kcal}^{\circ} \mathrm{semana}^{-1}\right)$. Además, el tiempo empleado en cada actividad se suma a un índice de tiempo total (h.semana-1). La segunda sección contiene preguntas para evaluar con rapidez la participación de un individuo en cinco dimensiones de actividad: actividad vigorosa, caminaron a ritmo tranquilo, moviendo de pie, de pie y sentado. Asignando un número a cada categoría de tal manera que la actividad vigorosa es 5; caminar es 4; en movimiento es 3; de pie es 2; por sesión es 1 . La puntuación de frecuencia y la duración se multiplican entre sí, y después se multiplica de nuevo por el factor de ponderación de cada dimensión para calcular un índice de para cada dimensión. La síntesis es la suma de los cinco índices individuales. Las respuestas sobre el cuestionario YALE permiten calcular 8 índices: el tiempo total dedicado a la semana en todas las actividades física, el gasto energético semanal en Kilocalorías por semana, cinco índices individuales para las dimensiones de actividad, y un índice de actividad global. Cada sujeto respondió de forma individual a las preguntas. La duración aproximada hasta completar el cuestionario fue de 20 minutos.

\section{Cuestionario Calidad de vida para enfermos renales Kidney Disease Quality of Life (KDQOL)}

Para valorar la calidad de vida del paciente con patología renal en tratamiento en diálisis se utilizó el Kidney Disease Quality of Life Short-Form (KDQOL$\mathrm{SF})^{18}$ en la versión para población española ${ }^{19}$. Se trata de una medida de autoinforme que reúne la doble condición de instrumento genérico y específico, lo que permite combinar en un solo cuestionario las ventajas de comparar (propia de los instrumentos genéricos) y la especificidad y sensibilidad al cambio (característica de los instrumentos específicos). Consta de 36 ítems de carácter genérico sobre el estado de salud (SF-36) distribuidos en 8 escalas: función física (10 ítems), rol físico (4 ítems), dolor (2 ítems), salud general (5 ítems), vitalidad (4 ítems), función social (2 ítems), rol emocional ( 3 ítems) y salud mental (5 ítems); cumplimentados con 43 ítems específicos de la enfermedad renal que conforman un total de 11 escalas: síntomas ( 12 ítems), efectos de la enfermedad renal (8 ítems), carga de la enfermedad renal (4 ítems), trabajo (2 ítems), función cognitiva ( 3 ítems), calidad de la interacción social (3 ítems), función sexual (2 ítems), sueño (4 ítems), apoyo social (2 ítems), estímulo del personal de diálisis (2 ítems) y satisfacción del paciente ( 1 ítem). En cada ítem la puntuación ob- 
tenida es transformada en una escala de 0 a 100 según el porcentaje alcanzado de la máxima puntuación posible, donde los valores más altos indican en todos los casos una mejor calidad de vida. La puntuación correspondiente a cada escala del KDQOL-SF se obtiene calculando la media de las puntuaciones directas transformadas.

\section{Senior Fitness Test (SFT)}

La Senior Fitness Test (SFT) es una batería de test que evalúa la capacidad física funcional, que se define como la capacidad de desarrollar las actividades de la vida diaria de forma segura e independiente y sin fatiga. En primer lugar los sujetos realizaron un calentamiento estandarizado de 10 minutos dirigido por un investigador, para posteriormente realizar los diferentes test de la batería SFT validada por Rikli y Jones ${ }^{20}$. Se llevaron a cabo 5 de las 6 pruebas del SFT: a) levantarse y sentarse de la silla para evaluar la fuerza del tren inferior; b) dinamometría manual que evalúa fuerza de extremidad superior (en este caso se sustituyó por el ejercicio de curl de bíceps para evitar problemas con la fístula en los pacientes hemodializados); c) sentarse y llegar al pie extendido para evaluar la flexibilidad del tren inferior; d) alcanzar las manos tras la espalda para medir la flexibilidad del tren su- perior, y e) una prueba de caminar durante 6 minutos para evaluar la resistencia aeróbica.

\section{Tratamiento estadístico}

Para el análisis de los datos se utilizó el programa estadístico SPSS, versión 15.0 para Windows (SPSS Inc, Chicago, Illinois, EEUU). Los resultados descriptivos se presentan como media \pm desviación estándar (DE). Tras realizar la prueba de normalidad de Kolmogorov Smirnov se determinó realizar pruebas no paramétricas. El nivel de significación se determinó en $p \leq 0,05$. Se estableció como variable independiente el grupo o tipo de tratamiento sustitutivo de Tx, HD y DP. Las variables dependientes estudiadas fueron condición física, calidad de vida y actividad física. En cada caso se estudió la influencia del grupo sobre las variables dependientes a través del test de Kuskall Wallis para $\mathrm{K}$ muestras independientes.

\section{Resultados}

Con respecto a los datos del cuestionario de cuantificación física YALE no hubo diferencias estadísticamente significativas, se muestran las medias de los sujetos para cada grupo en cada uno de los ítems (Tabla 1).

Tabla 1. Valores medios \pm DE de los diferentes derivados del cuestionario YALE de actividad física.

\begin{tabular}{|l|c|c|c|c|}
\hline & Tx & DP & HD & Nivel P \\
\hline Índice de tiempo total semana $(\mathrm{h} / \mathrm{sem})$ & $35,7 \pm 12,0$ & $17,3 \pm 9,8$ & $20,1 \pm 15,6$ & n.s \\
\hline Índice de gasto energético total semanal (Kcal/sem) & $9158,1 \pm 3269,7$ & $3753,3 \pm 2309,7$ & $4653,0 \pm 3279,0$ \\
\hline Índice de vigor & $23,1 \pm 19,9$ & $8,1 \pm 9,9$ & $10,0 \pm 12,2$ & n.s \\
\hline Índice de paseo & $16,7 \pm 20,3$ & $9,0 \pm 9,0$ & $10,6 \pm 13,0$ & n.s \\
\hline Índice de movimiento general & $10,0 \pm 4,8$ & $6,0 \pm 0,0$ & $7,0 \pm 4,0$ & n.s \\
\hline Índice de estar de pie & $7,2 \pm 3,2$ & $4,0 \pm 1,5$ & $6,0 \pm 3,0$ & n.s \\
\hline Índice de estar sentado & $2,1 \pm 0,8$ & $1,6 \pm 0,7$ & $2,6 \pm 0,8$ & n.s \\
\hline Índice de actividad total semanal & $59,4 \pm 29,4$ & $28,7 \pm 17,7$ & $36,3 \pm 23,8$ & n.s \\
\hline
\end{tabular}

Tx: Trasplantados, DP: Diálisis Peritoneal, HD: Hemodiálisis, n.s: no significativo.

Los sujetos trasplantados son los que realizan un mayor número de horas semanales de actividad física, y también conforman el grupo que más puntúa en todos los apartados a excepción del índice de estar sentado, en el que el grupo de HD obtiene la mejor puntuación.

En la Tabla 2 se presentan los resultados medios en los diferentes ítems del cuestionario SF36 de cada grupo. En los sujetos trasplantados la media más elevada se sitúa en la función social con 95,45 sobre los 100 puntos posibles. Respecto a la comparación inter-grupo, no se observan diferencias significativas en ninguno de los ítems (Tabla 2). 
[ Sonsoles Hernández Sánchez, et al ]

Valoración física, condición física y calidad de vida en pacientes con diferentes tratamientos renales sustitutivos

Tabla 2. Valores medios \pm DE de los 8 dominios del cuestionario SF-36.

\begin{tabular}{|l|c|c|c|c|}
\hline & Tx & DP & HD & Nivel P \\
\hline Función física & $86,3 \pm 16,8$ & $45,62 \pm 19,89$ & $53,33 \pm 23,59$ & n.s \\
\hline Rol físico & $23,8 \pm 2,5$ & $7,03 \pm 9,70$ & $8,33 \pm 10,94$ & n.s \\
\hline Dolor corporal & $84,9 \pm 14,8$ & $65,3 \pm 27,6$ & $48,0 \pm 36,6$ & n.s \\
\hline Salud general & $71,0 \pm 27,3$ & $34,2 \pm 22,1$ & $29,8 \pm 7,3$ & n.s \\
\hline Vitalidad & $77,0 \pm 22,8$ & $43,2 \pm 10,4$ & $60,4 \pm 26,7$ & n.s \\
\hline Función social & $95,4 \pm 10,1$ & $68,7 \pm 13,3$ & $58,3 \pm 35,0$ & n.s \\
Rol emocional & $22,7 \pm 7,5$ & $23,9 \pm 2,9$ & $16,6 \pm 12,9$ & n.s \\
\hline Salud mental & $73,3 \pm 31,9$ & $68,7 \pm 9,9$ & $56,3 \pm 15,7$ & n.s \\
\hline
\end{tabular}

Tx: Trasplantados, DP: Diálisis Peritoneal, HD: Hemodiálisis, n.s: no significativo.

En la Tabla 3 se presentan los resultados medios en los diferentes ítems del cuestionario KDQoL de cada grupo. Podemos observar que en los apartados referentes a la enfermedad los trasplantados puntúan con valores más elevados que los sujetos en HD y DP. Sin embargo, son los sujetos en diálisis los que obtienen mayor puntuación en lo referente a relaciones sociales y percepción de la calidad del personal de diálisis. (Tabla 3).

Tabla 3. Valores medios \pm DE de los indicadores del cuestionario KDQoL.

\begin{tabular}{|c|c|c|c|c|}
\hline & Tx & DP & HD & Nivel P \\
\hline Síntomas & $89,6 \pm 8,0$ & $72,1 \pm 17,8$ & $63,2 \pm 16,5$ & n.s \\
\hline Efectos de la ERC & $85,8 \pm 13,2$ & $53,9 \pm 20,9$ & $52,6 \pm 19,1$ & n.s \\
\hline Carga de la ERC & $85,2 \pm 13,1$ & $32,0 \pm 32,8$ & $31,2 \pm 25,6$ & n.s \\
\hline Situación laboral & $27,2 \pm 34,3$ & $6,2 \pm 17,6$ & $25,0 \pm 41,8$ & n.s \\
\hline Función cognitiva & $10,2 \pm 12,7$ & $24,1 \pm 19,4$ & $21,1 \pm 32,7$ & n.s \\
\hline Relaciones sociales & $10,9 \pm 16,3$ & $20,8 \pm 17,6$ & $28,8 \pm 31,1$ & n.s \\
\hline Función sexual & $55,6 \pm 40,0$ & $43,7 \pm 30,6$ & $31,2 \pm 18,9$ & n.s \\
\hline Sueño & $76,3 \pm 14,1$ & $63,7 \pm 15,3$ & $52,9 \pm 22,1$ & n.s \\
\hline Apoyo social & $84,8 \pm 30,2$ & $77,0 \pm 28,0$ & $63,8 \pm 26,7$ & n.s \\
\hline Personal diálisis & $60,2 \pm 48,9$ & $87,5 \pm 14,9$ & $63,7 \pm 17,2$ & n.s \\
\hline Satisfacción del paciente & $51,5 \pm 41,7$ & $87,5 \pm 14,7$ & $49,9 \pm 18,2$ & n.s \\
\hline
\end{tabular}

Tx: Trasplantados, DP: Diálisis Peritoneal, HD: Hemodiálisis, n.s: no significativo.

No existen diferencias significativas en ninguna de las pruebas físicas llevadas a cabo $(p \leq 0,05)$ (Tabla 4).

Tabla 4. Valores medios \pm DE de diferentes pruebas de condición física.

\begin{tabular}{l|c|c|c|c|}
\hline & Tx & DP & HD & Nivel P \\
\hline Dinamometría manual (Kg) & $33,9 \pm 6,3$ & $33,7 \pm 4,4$ & $32,7 \pm 6,3$ & n.s \\
\hline Sentarse y llegar al pie extendido (cm) & $(-0,2 \pm 12,9)$ & $(-9,0 \pm 8,4)$ & $(-13,0 \pm 11,0)$ & n.s \\
\hline Alcanzar las manos tras la espalda (cm) & $(-6,6 \pm 17,7)$ & $(-6,1 \pm 16,0)$ & $(-18,3 \pm 5,8)$ & n.s \\
\hline Levantarse y sentarse de la silla (repeticiones) & $10,3 \pm 1,9$ & $10,3 \pm 1,5$ & $11,6 \pm 3,6$ & n.s \\
Caminata 6 minutos & $493,4 \pm 72,2$ & $451,6 \pm 80,2$ & $405,7 \pm 135,6$ & n.s \\
\hline
\end{tabular}

Tx: Trasplantados, DP: Diálisis Peritoneal, HD: Hemodiálisis, n.s: no significativo. 


\section{Discusión y conclusiones}

El principal hallazgo del presente estudio es la ausencia de diferencias significativas entre los pacientes con tratamiento renal sustitutivo en las variables calidad de vida, actividad física, y condición física. Aunque no existen diferencias estadísticamente significativas en los parámetros que se obtienen del cuestionario YALE. Podemos observar que los sujetos del grupo Tx realizan más horas de actividad física semanal que los de los grupos HD o DP. Esto puede ser debido a que los pacientes trasplantados, por lo general, pueden realizar actividades de la vida diaria de una forma más independiente, ya que al no requerir dializarse pueden disponer de más tiempo libre y, por tanto, unas mayores posibilidades para llevar una vida activa. Aunque estadísticamente la actividad física no presente diferencias significativas entre los tres grupos, existe una ligera diferencia entre grupos, siendo los trasplantados los que mayor índice de tiempo total semana (ITT) (h-semana-1) realizan y por tanto los que mayor Índice de gasto energético total semanal (IGET) (Kcal·semana ${ }^{-1}$ ) tienen. Si comparamos los datos de este estudio con los varones españoles sanos $^{21}$ de entre 60 y 69 años podemos observar que el total de horas semanales de actividad física es similar, e incluso mayor en el grupo Tx que en la población sana, mientras que los grupos HD y DP muestran valores inferiores. En uno de los estudios pioneros con este tipo de cuestionario ${ }^{17}$, los valores de tiempo de actividad física total semanal en hombres fueron superiores a todos los grupos expuestos en este estudio.

Contrariamente a nuestra hipótesis los grupos no presentaron diferencias significativas, ni en el cuestionario general SF-36 ni en el específico KDQOL en ninguna de las escalas. Aunque una de las posibles limitaciones de este estudio fue el número de sujetos, una comparativa entre grupos puede ser una opción interesante para poder intervenir dentro del propio tratamiento en futuros estudios experimentales. EI primer estudio publicado que comparaba a estos tres grupos de población ${ }^{22}$, tampoco encontró diferencias significativas en las escalas del cuestionario SF36. Los estudios que comparan la calidad de vida de HD y DP muestran una mejor calidad de vida en DP23,24, mientras que en otros trabajos no existen diferencias significativas. Si comparamos los datos de este estudio con otro llevado a cabo en población turca, observamos que el grupo Tx percibe mejor la salud general que en el de Sayin et al. en el año $2007^{22}$, mientras que los grupos HD y DP la percibe peor. En un estudio desarrollado en España ${ }^{25}$, se establecieron los valores de referencia del cuestionario SF36 para población adulta de más de 60 años y los resultados medios de nuestro estudio fueron menores en todas las escalas del cuestionario en el grupo HD comparados con el trabajo citado anteriormente, mientras el grupo $\mathrm{Tx}$ puntuaron mayor que los valores de referencia en todas las escalas a excepción del rol emocional. Esto nos hace pensar que los pacientes trasplantados perciben su calidad de vida de una forma similar a la de la población general, lo que puede ser debido a que el trasplante les permite realizar una vida más independiente y autónoma que cuando estaban con tratamiento sustitutivo renal ${ }^{22}$. Con respecto al cuestionario específico de calidad de vida para enfermos renales (KDQOL), tampoco observamos diferencias significativas entre los 3 grupos de estudio en ninguna de las 11 escalas del cuestionario al igual que ocurre con el estudio realizado por Sayin en población turca ${ }^{22}$.

Hoy en día la ERC se considera una preocupación para la salud. Hay complicaciones como la uremia, anemia, miopatía y neuropatía que disminuyen la fuerza muscular, la capacidad cardiopulmonar y la calidad de vida ${ }^{26}$. Johansen ${ }^{27}$ investigó si los pacientes en hemodiálisis se diferencian de las personas sanas sedentarias. Este autor determinó que los hemodializados eran más sedentarios y que la disminución de actividad era proporcional al incremento de la edad. También indicó que la anemia y la debilidad muscular son factores clave en la disminución de la capacidad funcional. Los sujetos del grupo HD del presente estudio recorrieron una media de 405,75 metros, frente a los 451,68 y 493,40 metros de los grupos DP y TX, respectivamente. En cualquier caso, estas diferencias no alcanzaron significación estadística. Existen datos que valoran la resistencia aeróbica con la caminata de 6 minutos y observaron que los pacientes en hemodiálisis pudieron recorrer una distancia media de 445,22 metros, mientras el grupo control realizó 538 metros $^{28}$. En un estudio realizado en España con ERC en $\mathrm{HD}^{29}$ se aplicaron una serie de pruebas de dinamometría antes y después de un entrenamiento de fuerza-resistencia de 6 meses. Los resultados reflejan que los valores de fuerza son muy inferiores a los de nuestro estudio tanto antes como después de la aplicación del entrenamiento intradiálisis, con una media de 25 a 28 kilos. La media de nuestro estudio en sujetos en HD se sitúa en $32,70 \mathrm{~kg}$ por lo que los valores son mayores, pudiendo ser la causa el gran rango de edad que abarca el grupo de HD29. Por lo que se refiere a la flexibilidad, no ha sido un punto de interés preferente en la población objeto de estudio. Así, no hemos encontrado ningún trabajo específico con enfermos renales referentes a esta capacidad física. Aunque nuestros datos no muestran diferencias significativas 
entre los 3 grupos, el hecho de que la hemodiálisis suponga períodos de movilidad reducida puede abrir camino a nuevos estudios comparativos entre diferentes terapias de diálisis y la capacidad de flexibilidad de los sujetos.

En conclusión, en este trabajo presentamos los datos del primer estudio de cuantificación de nivel de actividad física, condición física y calidad de vida de enfermos renales crónicos llevado a cabo España. Nuestros datos indican que no existen diferencias significativas en niveles de actividad física, calidad de vida y condición física al comparar a sujetos con ERC sometidos a tres tratamientos sustitutivos diferentes. Futuros estudios deberían reforzar esta conclusión mediante el uso de herramientas diferentes y muestras mayores.

\section{Agradecimientos}

Agradecer al Departamento de Nefrología del Hospital Nuestra Señora de Sonsoles de Ávila, Hospital Clínico Universitario de Valladolid y Hospital de León su hospitalidad y colaboración para poder llevar a cabo este estudio, el cual ha estado financiado (en parte) por el Fondo Social Europeo y la Consejería de Educación de la Junta de Castilla y León bajo el Programa Operativo Castilla y León 2007-2013.

Recibido: 14 mayo 2015

Revisado: 16 mayo 2015

Modificado: 16 mayo 2015

Aceptado: 16 mayo 2015

\section{Bibliografía}

1. Kouidi, E., Grekas, D., Deligiannis, A., \& Tourkantonis, A. (2004). Outcomes of long-term exercise training in dialysis patients: comparison of two training programs. Clin Nephrol, 61 Suppl 1, S31-38.

2. K/DOQI clinical practice guidelines for chronic kidney disease: evaluation, classification, and stratification. (2002). Am J Kidney Dis, 39(2 Suppl 1), S1-266.

3. Johansen, K. L., Doyle, J., Sakkas, G. K., \& Kent-Braun, J. A. (2005). Neural and metabolic mechanisms of excessive muscle fatigue in maintenance hemodialysis patients. Am J Physiol Regul Integr Comp Physiol, 289(3), R805-813.

4. McIntyre, C. W., Selby, N. M., Sigrist, M., Pearce, L. E., Mercer, T. H., \& Naish, P. F. (2006). Patients receiving maintenance dialysis have more severe functionally significant skeletal muscle wasting than patients with dialysis-independent chronic kidney disease. Nephrol Dial Transplant, 21(8), 2210-2216.

5. Straub, C. K., Murphy, S. 0., \& Rosenblum, R. (2008). Exercise in the management of fatigue in patients on peritoneal dialysis. Nephrol Nurs J, 35(5), 469-475.

6. Vasilieva, I. A. (2006). Quality of life in chronic hemodialysis patients in Russia. Hemodial Int, 10(3), 274-278.

7. Painter, P., Carlson, L., Carey, S., Paul, S. M., \& Myll, J. (2000b). Physical functioning and health-related quality-of-life changes with exercise training in hemodialysis patients. Am J Kidney Dis, 35(3), 482492.

8. Deligiannis, A., Kouidi, E., Tassoulas, E., Gigis, P., Tourkantonis, A., \& Coats, A. (1999). Cardiac effects of exercise rehabilitation in hemodialysis patients. Int J Cardiol, 70(3), 253-266.

9. Konstantinidou, E., Koukouvou, G., Kouidi, E., Deligiannis, A., \& Tourkantonis, A. (2002). Exercise training in patients with end-stage renal disease on hemodialysis: comparison of three rehabilitation programs. J Rehabil Med, 34(1), 40-45.

10. Kouidi, E., Albani, M., Natsis, K., Megalopoulos, A., Gigis, P., Guiba-Tziampiri, 0., et al. (1998). The effects of exercise training on muscle atrophy in haemodialysis patients. Nephrol Dial Transplant, 13(3), 685-699.

11. Molsted, S., Eidemak, I., Sorensen, H.T., \& Kristensen, J. H. (2004). Five months of physical exercise in hemodialysis patients: effects on aerobic capacity, physical function and self-rated health. Nephron Clin Pract, 96(3), c76-81. 
12. Headley, S., Germain, M., Mailloux, P., Mulhern, J., Ashworth, B., Burris, J., et al. (2002). Resistance training improves strength and functional measures in patients with end-stage renal disease. Am J Kidney Dis, 40(2), 355-364.

13. Oh-Park, M., Fast, A., Gopal, S., Lynn, R., Frei, G., Drenth, R., et al. (2002). Exercise for the dialyzed: aerobic and strength training during hemodialysis. Am J Phys Med Rehabil, 81(11), 814-821.

14. Painter, P., Carlson, L., Carey, S., Paul, S. M., \& Myll, J. (2000a). Low-functioning hemodialysis patients improve with exercise training. Am $J$ Kidney Dis, 36(3), 600-608.

15. Painter, P., Moore, G., Carlson, L., Paul, S., Myll, J., Phillips, W., et al. (2002). Effects of exercise training plus normalization of hematocrit on exercise capacity and health-related quality of life. Am J Kidney Dis, 39(2), 257-265.

16. Dipietro, L., Caspersen, C. J., Ostfeld, A. M., \& Nadel, E. R. (1993). A survey for assessing physical activity among older adults. Med Sci Sports Exerc, 25(5), 628-642.

17. De Abajo, S., Larriba, R., \& Marquez, S. (2001). Validity and reliability of the Yale Physical Activity Survey in Spanish elderly. J Sports Med Phys Fitness, 41(4), 479-485.

18. Hays, R. D., Kallich, J. D., Mapes, D. L., Coons, S. J., \& Carter, W. B. (1994). Development of the kidney disease quality of life (KDQOL) instrument. Qual Life Res, 3(5), 329-338.

19. García-López, F. J., López-Revuelta, K., Álvaro, F., Prieto, L., \& Alonso, J. (1999). Spanish validation of the short form of the Kidney Disease Quality of Life Instrument. Qual Life Res 8, 602.

20. Rikli, R. E., \& Jones, C. J. (1999). Development and validation of a functional fitness test for communityresiding older adults. J. Aging Phys. Act. , 17, 127159.

21. Garatachea, N., Molinero, 0., Martinez-Garcia, R., Jimenez-Jimenez, R., Gonzalez-Gallego, J., \& Marquez, S. (2009). Feelings of well being in elderly people: relationship to physical activity and physical function. Arch Gerontol Geriatr, 48(3), 306-312.

22. Sayin, A., Mutluay, R., \& Sindel, S. (2007). Quality of life in hemodialysis, peritoneal dialysis, and transplantation patients. Transplant Proc, 39(10), 3047-3053.
23. Carmichael, P., Popoola, J., John, I., Stevens, P. E., \& Carmichael, A. R. (2000). Assessment of quality of life in a single centre dialysis population using the KDQOL-SF questionnaire. Qual L ife Res, 9(2), 195205.

24. Majkowicz, M., Afeltowicz, Z., LichodziejewskaNiemierko, M., Debska-Slizien, A., \& Rutkowski, B. (2000). Comparison of the quality of life in hemodialysed (HD) and peritoneally dialysed (CAPD) patients using the EORTC QLQ-C30 questionnaire. Int J Artif Organs, 23(7), 423-428.

25. Lopez-Garcia, E., Banegas, J. R., Graciani PerezRegadera, A., Gutierrez-Fisac, J. L., Alonso, J., \& Rodriguez-Artalejo, F. (2003). [Population-based reference values for the Spanish version of the SF36 Health Survey in the elderly]. Med Clin (Barc), 120(15), 568-573.

26. Yurdalan, S. U., Kondu, S., \& Malkoc, M. (2007). Assessment of health-related fitness in the patients with end-stage renal disease on hemodialysis: using Eurofit Test Battery. Ren Fail, 29(8), 955-960.

27. Johansen, K. L. (1999). Physical functioning and exercise capacity in patients on dialysis. Adv Ren Replace Ther, 6(2), 141-148.

28. Chen, Y. C., Chen, H. H., Yeh, J. C., \& Chen, S. Y. (2000). Body composition in hemodialysis patients-is it different from that of normal subjects? Clin Nephrol, 53(4), 291-295.

29. Segura-Orti, E., Kouidi, E., \& Lison, J. F. (2009). Effect of resistance exercise during hemodialysis on physical function and quality of life: randomized controlled trial. Clin Nephrol, 71(5), 527-537. 